\title{
Fractional Order PID Design using the Taguchi Method
}

\author{
Somayeh Norouzi, Alireza Akbarzadeh \\ Mechanical Engineering Department, Ferdowsi University of Mashhad, Mashhad, Iran \\ Center of Excellence on Soft Computing and Intelligent Information Processing (SCIP), \\ Ferdowsi University of Mashhad, Iran
}

\begin{tabular}{l} 
Article Info \\
Article history: \\
Received Apr 12, 2015 \\
Revised Jul 26, 2015 \\
Accepted Aug 10, 2015 \\
\hline
\end{tabular}

Keyword:

Fractional Order

PID Design

Taguchi Method

Ziegler-Nichols (Z-N) method

\begin{abstract}
This paper presents a gain-tuning scheme for Fractional order PID control systems using the Taguchi zmethod. A prismatic series elastic actuator is selected as an experimental set-up. An optimal controller gains has been obtained through a series of experiments suggested by the Taguchi method. Four stages of tuning are performed in order to accurately tune the controller gains. It is shown that when performance of the proposed controller is compared with two additional controllers: a traditional FOPID tuned with Ziegler-Nichols (Z-N) method and a PID tunned with genetic algorithm, a $94 \%$ and $84 \%$ improvements in position error is observed, respectively.
\end{abstract}

Copyright $(2015$ Institute of Advanced Engineering and Science. All rights reserved.

\section{Corresponding Author:}

Somayeh Norouzi,

Mechanical Engineering Department,

Ferdowsi University of Mashhad,

Mashhad, Iran.

Email: somaye_noroozi@yahoo.com

\section{INTRODUCTION}

The Fractional Order Calculus (FOC) constitutes the branch of mathematics that deals with derivatives and integrals from non-integer orders. Although FOC is a more than 300 years old topic, its great consequences in contemporary theoretical and experimental research including in the area of control theory have been widely discussed relatively recently.

Fractional order proportional-integral-derivative (FOPID) controllers have received a considerable attention in the last years both from academic and industrial point of view. As there are five parameters to select instead of three in standard PID type controllers, these controllers provide more flexibility in the controller design. However, this also implies that tuning of the controller can be much more complex. During the last years, several techniques have been suggested for tuning of the controller gains.

For the first time the concept of FOPID controller and its better performance in comparison with the classical PID controller was introduced by Podlunbny [1] in 1997. Vinagre [2] in 2000, studied a frequency domain approach by using FOPID controllers. An optimization method where the parameters of the FOPID are tuned with predefined methods is presented in 2004 by Monje et al. [3]. In 2006, Valerio and Costa [4] proposed Zigler-Nichols tuning rules for FOPID. In 2009, an optimization method with particle swarm optimization was reported by Zamani et al [5]. A tuning and auto tuning method for FOPID controllers based on gain crossover frequency and phase margine was proposed in 2007 by Monje et al [6].

Lee and Kim [7] in 2000, studied gain-tuning for multi-axis PID control system using the Taguchi method. In 2002, Ryckebusch [8] used Taguchi method for tuning PID controller parameters in a multivariable plant. In Ryckebusch's research two PID controllers were tuned simultaneously. Another Taguchi based tuning method for two-degree-of freedom proportional-integral controller was reported by 
Alfaro et al. [9] in 2010. Self-tuning mechanism of PID controller based on Taguchi method was reported in 2010 by Santhakumar and Asoka [10].

A series elastic actuator (SEA) is selected as an experimental set-up, and is shown in Figure 1.
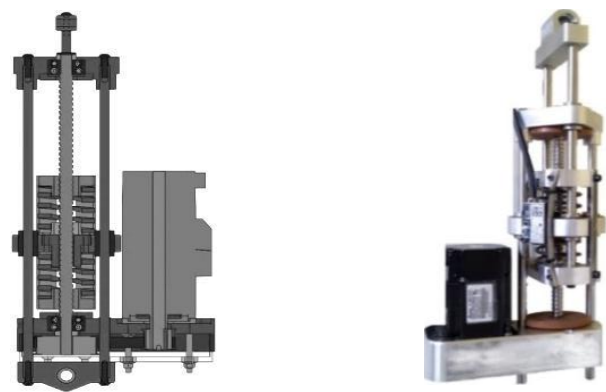

Figure 1. Series Elastic Actuator

SEAs or variable stiffness actuators include an elastic element, e.g., a linear spring, placed between the gear motor and the load (Figure 1). The additional element, the elastic element, is used to elastically decouple the actuator from the load and improves tolerance to mechanical shocks. If proper stiffness is selected according to the target task, the spring can also protect the motor and gearbox in the case of unwanted collisions in the output links [11]. This ability causes that SEAs are increasingly applied to humanassisted robotic systems. Human assistive robots, i.e., systems that assist human motions with actuation capabilities, have been intensively developed in recent years [11,12]. To effectively assist human motions actuators with capability of generating large torques are required. In human-assistance scenarios, the actuator is connected to single or multiple joints of human in order to provide assistive torques. As there is a connection between the patient and the system, meeting precise control demands is also very important and required. With increasingly usage of series elastic actuators in human assistive robots precise control of these actuators draws a lot of attention in recent years. Control of SEA with PID-type controller proposed by Au [13] in 2006. Kong [14] in 2009 presented force control of a rotary series elastic actuator. A study on force control of a SEA with rotary spring by using PID controller was reported by Taylor [15] in 2011. Hutter [16] in 2011 studied fast position control of high compliant series elastic actuator. Misgeld et al [17] in 2014 studied robust control of adjustable compliant actuators.

This paper presents the use of Taguchi method in order to tuning FOPID controller gains. The outline of this paper is as follows. The Taguchi method is introduced in section 3 and the procedure of experimentation and results are presented in section 4. Concluding discussion is given in section 5.

\subsection{Modeling}

A simple model for an actuator with a series elastic element is shown in Figure 2.

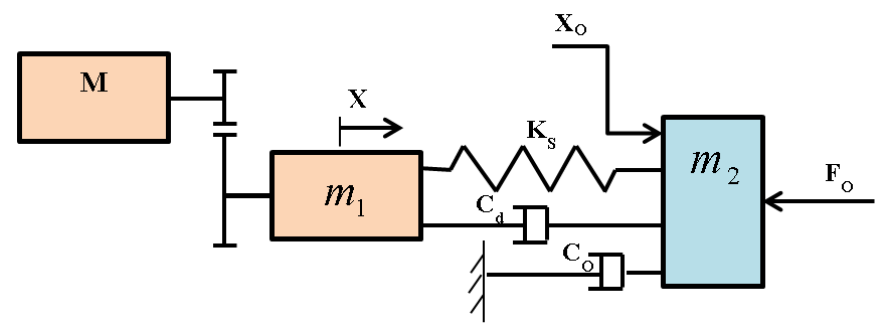

Figure 2. A Series Elastic Actuator Model

The notations represent: motor: $M$, the mass of nut and ball screw: $m_{l}$, the spring stiffness: $K_{s}$, the coulomb friction constant of guides: $C_{d}$, the the coulomb friction constant between load and fixed part of SEA: $C_{o}$, the load mass: $\mathrm{m}_{2}$, the movement of load: $X_{o}$, the movement of shaft: $X$ and the output force: $F_{o}$. The friction is assumed to be negligible [ $\left.{ }^{\mathrm{i}}\right]$, and then control of a simple model is proposed. 
From the diagram by applying Newoton's law the relations between $X_{o}, F_{m}$ (motor force) and $F_{o}$ can be written as below (Eq. 1 and 2),

$$
\begin{aligned}
& m_{1} \ddot{x}=F_{m}-k_{s}\left(x-x_{o}\right) \\
& F_{o}=k_{s}\left(x-x_{o}\right)
\end{aligned}
$$

By taking Laplace transform, an expression relating $F_{m}$ and $F_{o}$ can be found as Eq. (3),

$$
F_{o}(s)=\left(\frac{k_{s}}{m_{1} s^{2}+k_{s}}\right) F_{m}(s)-\left(\frac{k_{s} m_{1} s^{2}}{m_{1} s^{2}+k_{s}}\right) X_{o}(s)
$$

Eq. (3) shows how $F_{m}$ needs to vary to give a desired output force while the output load is moving. The block diagram of the system is shown in Figure 3.

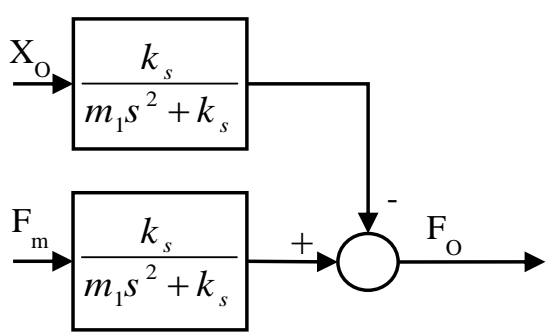

Figure 3. The block diagram of the system

\subsection{Control}

Figure 4. illustrates a drawing of the proposed control scheme. It includes the feed forward terms and the PID control loop.

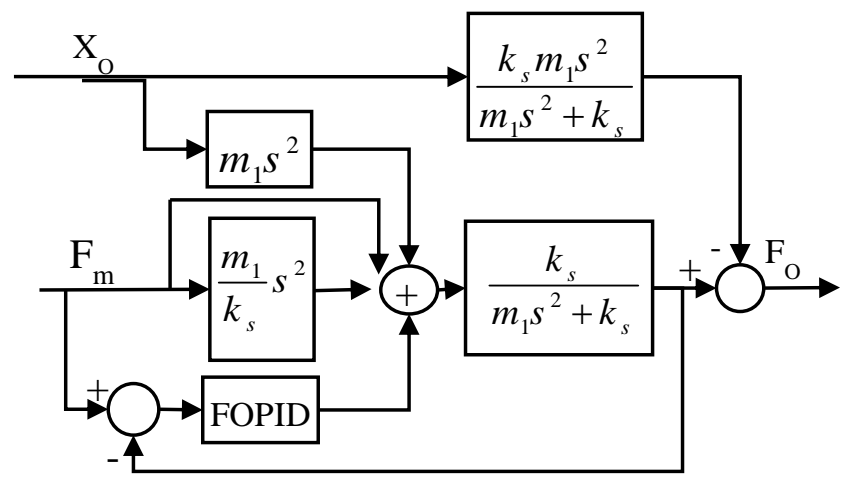

Figure 4. The proposed control system

The feed forward gains are obtained from the Equation 4,

$$
F_{m}(s)=F_{o}(s)\left(\frac{m_{1}}{k_{s}} s^{2}+1\right)+m_{1} s^{2} X_{o}(s)
$$

The value of the parameters in the above transfer functions are listed in the Table 1 
Table 1. System parameters

\begin{tabular}{lcc}
\hline Parameter & $k_{s}(\mathrm{~N} / \mathrm{m})$ & $m_{1}(\mathrm{~kg})$ \\
\hline value & 60 & 0.7 \\
\hline
\end{tabular}

\subsubsection{Fractional Calculus}

The fundamental operator ${ }_{a} D_{t}^{r} f(t)$, is a combined differentiation-integration operator commonly used in fractional calculus. The ${ }_{a} D_{t}^{r}$ is called the fractional derivative/ integral of order $r$ with respect to variable $t$ and with the starting point $a$. It is defined as Eq. $5\left[^{5}\right]$,

${ }_{a} D_{t}^{r}= \begin{cases}\frac{d^{r}}{d t^{r}} & r>0, \\ 1 & r=0, \\ \int_{a}^{t}(d \tau)^{-r} & r<0,\end{cases}$

The three definitions most frequently used for the fractional derivatives are the Grunwald-Letnikov (GL), the Riemen-Liouville (RL) and the Caputo definition [ii]. The GL fractional derivative which is used here has the following form (Eq. 6),

$$
{ }_{a} D_{t}^{r} f(t)=\lim _{h \rightarrow 0} h^{-r} \sum_{j=0}^{\left[\frac{t-a}{h}\right]}(-1)^{j}\left(\begin{array}{l}
r \\
j
\end{array}\right) f(t-j h)
$$

where [.] means the integer part.

For the computation of the coefficients to obtain the numerical solution, when $\alpha$ has a fixed value of derivative order, the following recursive formula (Eq. 7 and 8) can be used [iii],

${ }_{a} D_{t}^{r} f(t) \approx \lim _{h \rightarrow 0} h^{-r} \sum_{j=0}^{\left[\frac{t-a}{h}\right]} \omega_{j}^{(\alpha)} f(t-j h)$

where,

$$
\omega_{0}^{(\alpha)}=1, \quad \omega_{j}^{(\alpha)}=\left(1-\frac{\alpha+1}{j}\right) \omega_{j-1}^{(\alpha)}
$$

Laplace transform of non-integer order derivatives is a fundamental tool in design of a FOPID controller. In the case of Laplace transform, there is no big difference with respect to the classical case. Besides, Inverse Laplace transformation is necessary for time domain representation of the system. The Laplace transformation is described by the following equation (Eq. 9) [20],

$$
L\left[{ }_{a} D_{t}^{\alpha} f(t)\right]=s^{\alpha} L[f(t)]
$$

\subsubsection{Fractional order PID (FOPID) Controller}

The integral-differential equation defining the control action of a fractional order PID $\left(P I^{-\lambda} D^{\mu}\right)$ controller is given by (Eq. 10)

$u(t)=K_{p} e(t)+K_{i} D^{-\lambda} e(t)+K_{d} D^{\mu} e(t)$

where $e(t)$ is the error signal of a tracking system, $u(t)$ is the control signal, $\lambda$ and $\delta$ are positive real numbers; $K_{p}$ is the proportional gain, $K_{i}$ the integration constant and $K_{d}$ the differentiation constant. 
$P I^{-\lambda} D^{\mu}$ Controller, also known as $P I^{\lambda} D^{\delta}$ controller, was studied in time domain in $\left[{ }^{\mathrm{iv}}\right]$ and in frequency domain in $\left.{ }^{\mathrm{v}}\right]$.

\subsection{The Taguchi Method}

The Taguchi method based on the fractional factorial experiment, divides the independents parameters into design parameters and noise parameters. Design parameters are those for which the designer selects values as a part of the design process and noise parameters model uncertainty in the design. In the design process, the design parameter values are chosen such that the design goals are maximized despite the noise parameter actions. This means that a robust tuning technique using the Taguchi method would enable regulators not only to reduce control errors but also to decrease variations in those values while remaining insensitive to changes in system dynamics and variations in operating points. By applying this approach researchers can significantly reduce the time required for experimental investigations.

In this paper, experiments to tune a total of 5 position controller gains of a series elastic actuator are presented. The "Design of Experiment" using the Taguchi method is briefly outlined below $\left[^{7}\right]$ :

1) Identifying the objectives: in the first step of the Taguchi method, identifying a specific objective is important. In this paper, the objective is a robust tuning of controller gains for minimizing position error.

2) Determining the quality characteristic: the Taguchi method classifies quality characteristics into one of three types: nominal-the-best, smaller-the-better and larger-the-better. In this study, the smaller the position error, the better performance.

3) Selecting the controllable factors and noise factors: the selection of factors to be tested for their influence on the quality characteristic is one of the most important procedures. Careless selection of controllable factors and noise factors can lead to false conclusions and can require experiments to be repeated. After selecting factors, their desired number of levels is determined. In this paper, controller gains are used as the controllable factors and load position is used as the noise factor. The number of levels for controller gains is three and for load position is also three. The next step is to assign a physical value to each level of controllable factors.

4) Selecting an orthogonal array: The full factorial experiment requires the testing of all combinations of the factor levels under study. For example, a study involving 5 factors at three levels each would require $3^{5}=243$ experiments. Orthogonal arrays produce smaller, less costly experiments. Using an $L\left(3^{5}\right)$ orthogonal array, for example, a study involving 5 factors at three levels can be conducted with only 27 experiments. Besides being efficient, the procedures for using orthogonal arrays are straightforward and easy to use. In this paper, in order to reduce the number of experiments an $L_{16}\left(4^{5}\right)$ orthogonal array is selected. This array implies only 16 experiments on condition that one level of each factor would then be "empty" in the array.

5) Conducting the experiment and analysis: Conducting the experiment includes the execution of the experiment as developed in the planning and design phases. The analysis phase of experimentation relates to calculations for converting raw data into the representative signal-to-noise ratio (SNR ratio). As a measurement tool for determining robustness, the SNR ratio is an essential component to optimal parameter design. By including the impact of noise factors on the process, the $\mathrm{S} / \mathrm{N}$ ratio can be adopted as the index of the system's ability to perform well regardless of the noise. By successfully applying this concept to experimentation, it is possible to determine the controller gain settings that can produce the minimum position errors while maximizing the signal to noise ratio. For this partial-fractional experiment design, the $\mathrm{S} / \mathrm{N}$ ratio in case of smaller-the-better quality characteristic can be written as (Eq. 12 ) $\left.{ }^{8}\right]$ :

$$
\begin{aligned}
& I T A E=\int t|e(t)| d t \\
& \mathrm{SNR}=-10 \log I T A E
\end{aligned}
$$

In this paper, the criterion of performance is the integral of time weighted absolute error (ITAE) between the corresponding outputs and set point. The ITAE criteria for FOPID controller tuning provides closed loop response with low overshoot and fast response [23].

Since the Taguchi method reduces the number of experiments over the full-factorial approach, it is useful to use the statistical analysis of experiments, called analysis of variance (ANOVA), to provide levels of confidence in the results. Moreover, analysis of variance identifies and ranks variables that affect the variance of the output signal. This ANOVA is one of the main steps in using the Taguchi method. 


\subsection{Experiments}

The initial values for the experimentation were found with the traditional Ziegler-Nichols (Z-N) method [24]. Sustained oscillations are observed quite easily and the Z-N formula gives initial values for proportional, integrative and derivative parameters as shown in table 2.

Table 2. The initial values for FOPID controller obtained from Z-N method

\begin{tabular}{lccccc}
\hline Parameter & $K_{p}$ & $K_{i}$ & $\lambda$ & $K_{d}$ & $\mu$ \\
\hline Value: & 0.6 & 0 & - & 3.0 & 0.3 \\
\hline
\end{tabular}

The time response of the controlled system which is tuned by Z-N tuning technique is shown in Fig. ( 5).

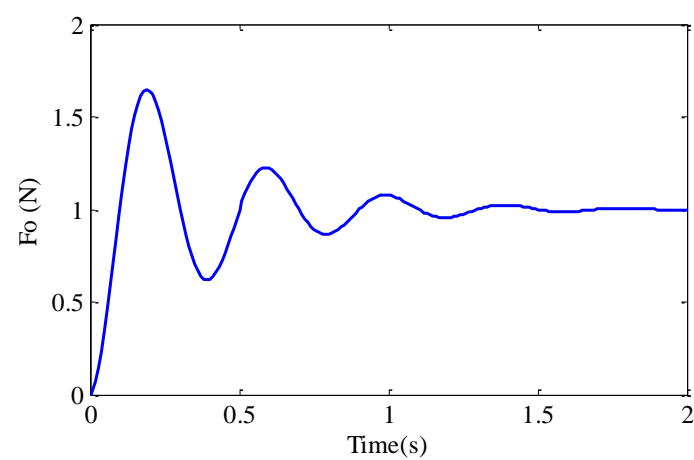

Figure 5. Closed loop response with a FOPID controller tuned by Z-N technique

The first test with these initial values gives error and SNR equal to 17.305 and -48.81 respectively. From table 2 it is found that the initial value for fractional order integrator gain is obtained equal to zero, this corresponds to FOPD controller, then the number of parameters in FOPID controller reduces to 3 parameters. Again there is a need to determine the appropriate orthogonal array. A $L_{9}\left(3^{3}\right)$ is determined at this stage. The experimental layout is shown in table 3 . The nine rows of this matrix represent the experiments to be conducted. The three columns corresponds to three parameters or factors (respectively: kp, kd, $\mu$ ).

Table 3. Experimental layout based on $L_{9}\left(3^{3}\right)$

\begin{tabular}{cccc}
\hline \# of Runs & $K_{p}$ & $K_{d}$ & $\mu$ \\
\hline 1 & 1 & 1 & 1 \\
2 & 1 & 2 & 2 \\
3 & 1 & 3 & 3 \\
4 & 2 & 1 & 2 \\
5 & 2 & 2 & 3 \\
6 & 2 & 3 & 1 \\
7 & 3 & 1 & 3 \\
8 & 3 & 2 & 1 \\
9 & 3 & 3 & 2 \\
\hline
\end{tabular}

The variation range of parameters is shown in the table 4 .

Table 4. The variation range of parameters

\begin{tabular}{cccc}
\hline Parameters & $1^{\text {st }}$ level & $2^{\text {nd }}$ level & $3^{\text {rd }}$ evel \\
\hline$K_{p}$ & 0.5 & 1 & 5 \\
$K_{d}$ & 0.3 & 3 & 9 \\
$\mu$ & 0.3 & 0.6 & 1.3 \\
\hline
\end{tabular}


The experiments were carried out for each level and the errors were calculated for each experiment. Then the SNR were calculated for each experiment based on smaller the better characteristics. The sum of SNR was calculated for each parameter and at each level as shown in Table 5.

Table 5. Orthogonal array for FOPD controller

\begin{tabular}{cccccc}
\hline \# of Runs & $K_{p}$ & $K_{d}$ & $\mu$ & ISE & SNR \\
\hline 1 & 1 & 1 & 1 & 154.63 & -43.78 \\
2 & 1 & 2 & 2 & 11.55 & -21.25 \\
3 & 1 & 3 & 3 & 24.79 & -27.88 \\
4 & 2 & 1 & 2 & 134.50 & -42.57 \\
5 & 2 & 2 & 3 & 32.68 & -30.28 \\
6 & 2 & 3 & 1 & 2.66 & -8.49 \\
7 & 3 & 1 & 3 & 54.83 & -34.78 \\
8 & 3 & 2 & 1 & 13.18 & -22.39 \\
9 & 3 & $\mathbf{3}$ & $\mathbf{2}$ & $\mathbf{1 . 8 6}$ & $\mathbf{- 5 . 3 8}$ \\
\hline
\end{tabular}

By plotting the average response value for each factor level (Figure 9), relative comparisons of the slope between points plotted can be made.

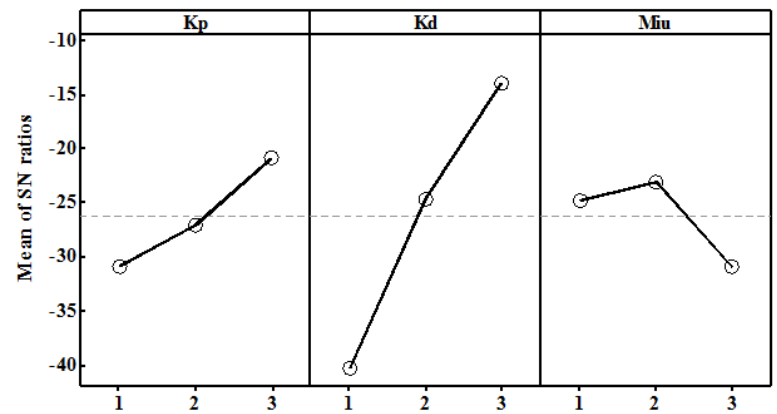

Figure 6 . Main effects plot for SN ratios

The response graph indicates that factor $K_{d}$ has the strongest effect, whereas $K_{p}$ and $\mu$ have weak effect. In other words, the variations of $K_{d}$ value is more sensitive to the $\mathrm{S} / \mathrm{N}$ ratio variation than those of $K_{p}$ and $\mu$. As in any signal-to-noise analysis, the greatest $\mathrm{S} / \mathrm{N}$ ratio is recommended, based on table 5, The optimum level of each parameter i.e. the level that results in a maximum $\mathrm{S} / \mathrm{N}$ ratio are those in experiment number 1 , that is all variables at their first level. From the $1^{\text {st }}$ stage of tuning of controller's parameters with Taguchi method the error is reduced by $89 \%$. However, the reduction of error is not significant to prevent another stage of tuning.

Considering Figure 7, It is observed from that the preferred levels for $K_{d}$ and $\mu$ are set at level 3 and the $\mathrm{S} / \mathrm{N}$ ratio decreases continually with each controller gain. This means that values for $K_{d}$ and $\mu$ that can maximize the $\mathrm{S} / \mathrm{N}$ ratio may be larger than the preferred levels. Accordingly, based on the preferred levels at stage 1, controller gains should be tuned again, in stage 2 .

Table 6. Levels of controller gains at stages 2 to 4

\begin{tabular}{cccccccccc}
\hline & \multicolumn{3}{c}{ Stage 2} & \multicolumn{5}{c}{ Stage 3 } & \multicolumn{3}{c}{ Stage 4 } \\
\hline Parameters & $\begin{array}{c}1^{\text {st }} \\
\text { level }\end{array}$ & $\begin{array}{c}2^{\text {nd }} \\
\text { level }\end{array}$ & $\begin{array}{c}3^{\text {rd }} \\
\text { level }\end{array}$ & $\begin{array}{c}1^{\text {st }} \\
\text { level }\end{array}$ & $\begin{array}{c}2^{\text {nd }} \\
\text { level }\end{array}$ & $3^{\text {rd }}$ level & $\begin{array}{c}1^{\text {st }} \\
\text { level }\end{array}$ & $\begin{array}{c}2^{\text {nd }} \\
\text { level }\end{array}$ & $3^{\text {rd }}$ level \\
\hline$K_{p}$ & 4 & 5 & 8 & 4.9 & 5 & 5.2 & 4.9 & 5 & 5.2 \\
$K_{d}$ & 8 & 9 & 12 & 8.9 & 9 & 9.2 & 8.9 & 9 & 9.2 \\
$\mu$ & 0.5 & 0.6 & 0.7 & 0.7 & 0.9 & 1.1 & 0.7 & 0.9 & 1.1 \\
\hline
\end{tabular}

Response graphs for four stages are shown in table 7. 
Table 7. Errors and SNRs for stages 2 to 4

\begin{tabular}{cccccccccc}
\hline & & & \multicolumn{4}{c}{ Stage 2} & \multicolumn{2}{c}{ Stage 3 } & \multicolumn{2}{c}{ Stage 4 } \\
\hline \# of Runs & $K_{p}$ & $K_{d}$ & $\mu$ & ITAE & SNR & ITAE & SNR & ITAE & SNR \\
\hline 1 & 1 & 1 & 1 & 2.14 & -6.62 & 1.44 & -3.15 & 1.18 & -1.45 \\
2 & 1 & 2 & 2 & 1.49 & -3.46 & $\mathbf{1 . 0 0}$ & $\mathbf{0 . 0 0}$ & $\mathbf{1 . 0 0}$ & $\mathbf{0 . 0 0}$ \\
3 & 1 & 3 & 3 & 1.60 & -4.06 & 1.23 & -2.22 & 1.16 & -1.30 \\
4 & 2 & 1 & 2 & 1.95 & -5.80 & $\mathbf{1 . 0 0}$ & $\mathbf{0 . 0 0}$ & $\mathbf{1 . 0 0}$ & $\mathbf{0 . 0 0}$ \\
5 & 2 & 2 & 3 & $\mathbf{1 . 4 4}$ & $\mathbf{- 3 . 1 9}$ & 1.27 & -2.12 & 1.15 & -1.20 \\
6 & 2 & 3 & 1 & 2.47 & -7.87 & 1.43 & -3.13 & 1.12 & -0.97 \\
7 & 3 & 1 & 3 & 2.04 & -6.21 & 1.26 & -2.01 & 1.13 & -1.09 \\
8 & 3 & 2 & 1 & 3.34 & -10.47 & 1.46 & -3.27 & 1.29 & -2.24 \\
9 & 3 & 3 & 2 & 6.37 & -16.09 & $\mathbf{1 . 0 0}$ & $\mathbf{0 . 0 0}$ & $\mathbf{1 . 0 0}$ & $\mathbf{0 . 0 0}$ \\
\hline
\end{tabular}

In stage 2, the experimental procedure is the same as for gain tuning at stage 1 except for the levels of controller gains. After conducting gain tuning at stage 2, if making the $\mathrm{S} / \mathrm{N}$ ratio greater is feasible, additional gain tuning is performed. By following these methods, gain tuning using the Taguchi method is completed at stage 4 . Table 6 represents the levels of controller gains selected in stages 2,3 and 4 . The preferred levels for controller gains at each stage are indicated in bold type in Table 6 .
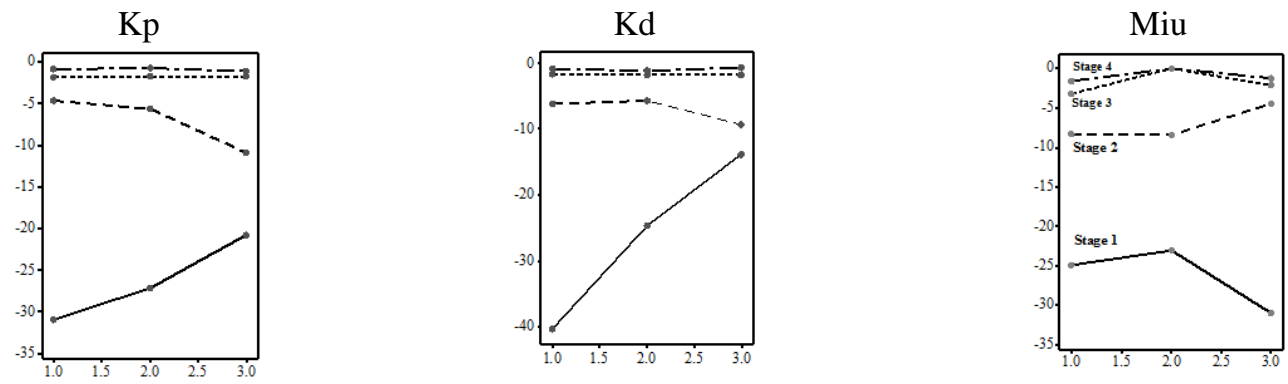

Figure 7. Main effects plots for SN ratios for four stages

Response graphs at stage 4 in Figure 7 shows that $\mathrm{S} / \mathrm{N}$ ratios for $K_{p}, K_{d}$ and $\mu$ do not increase any further and the preferred levels are first levels for $K_{p}$ and $K_{d}$ and second level for $\mu$. This means that their optimal levels exist around these values. Controller gains are finally tuned at stage 4 .

Closed loop response for the three system controllers (FOPID controller tuned by Z-N technique, final FOPID controller tuned by Taguchi method and PID controller tuned by genetic algorithm presented by Hussain In $\left[{ }^{\mathrm{vi}}\right]$ ) are shown in Figure 8.

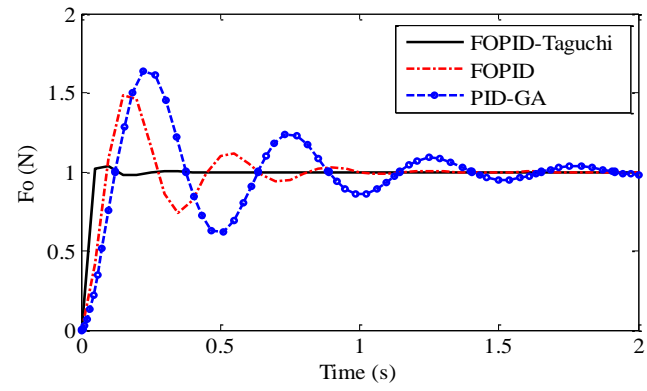

Figure 8. Closed loop response with a FOPID controller tuned by Z-N technique (Dash line) by Taguchi method (Solid line) 
Considering Figure 8, it can be observed that the FOPID tuned by Taguchi method gives better time domain performance with respect to the regular FOPID controller and the proposed method in [25], especially for maximum overshoot and settling time. With this best combination of gains the SNR increased to about $100 \%$. Results are confirmed by the ANOVA analysis with the percentage contribution shown in Table 8. The main purpose of this analysis is to estimate the effects that each factor has on the final results.

Table 8. Percentage Contribution

\begin{tabular}{ccc}
\hline Controller Parameter & $\begin{array}{c}\text { Percentage } \\
\text { contribution }\end{array}$ & P-Value \\
\hline$K_{p}$ & $5.40 \%$ & 0.62 \\
$K_{d}$ & $5.97 \%$ & 0.59 \\
$\mu$ & $79.81 \%$ & 0.09 \\
ANOVA Error & $8.81 \%$ & \\
\hline
\end{tabular}

ANOVA analysis shows that $\mu$ parameter has the biggest percentage contribution. It confirms results of figure 7 which illustrates that SNR values for the levels of $\mu$ differ significantly at stage 4 . It is important to note that the error contribution computed with ANOVA gives an idea of the confidence in the results. P-values which are shown in second column also confirms that at stage 4 variations of parameters $K_{p}$ and $K_{d}$ do not have important effects on improvement of SNR value.

\section{CONCLUSION}

This paper presented dynamic modeling and control of a series elastic actuator. The capability of generating large forces and low friction are why this actuator is increasingly used in the human-assistive robotic systems. Due to having the human in the loop, the actuator requires precise control. A fractional PID controller known for its improved performance due to having additional degrees of freedom than the classical PID is used for the control. Using fractional order PID controller, a significant improvement in position error was obtained. Tuning of the FOPID controller gains, for the first time, was performed using a new gain tuning technique based on the Taguchi method. Controller gains were tuned through four stages.

An orthogonal array $L_{9}\left(3^{3}\right)$ with four repitions were used. A $94 \%$ improvement in position error and about $100 \%$ improvement in signal to noise ratio in comparison with traditional FOPID controller were achieved. Comparison of the results of the proposed method with results obtained from an optimized PID controller tuned by genetic algorithm illustrated that FOPID tuned by Taguchi method gave better time domain performance, especially in terms of settling time and maximum overshoot. As settling time and maximum overshoot are two of more important charachteristics of the response of human assistive robots, then the results of this paper can potentially enable exo-skeleeton applications requiring faster and more accurate motion.

\section{REFERENCES}

[1] Podlubny, I., Dorcak, L., Kostial, I., "On Fractional Derivatives, Fractional-Order Dynamic Systems and PID controllers", Proceedings of the 36th Conference on Decision \& Control, San Diego, California, USA, (1997).

[2] Vinagre, B. M., Podlubny, I., Dorcak, L., Feliu, V., "On Fractional PID Controllers: A Frequency Domain Approach", Proceedings of IFAC Workshop on Digital Control: Past, Present and Future of PID Control. Terrasa, Spain, pp. 53-58, (2000).

[3] Monje, C.A., Vinagre, B.M. , Chen, Y.Q., Feliu, V., Lanusse, P., Sabatier, J., "Proposals for fractional $P I^{\lambda} D^{\mu}$ tuning”, Proceedings of Fractional Differentiation and its Applications, Bordeaux, (2004).

[4] Vale'rio, D., Costa, J.S., "Tuning of fractional PID controllers with Ziegler-Nichols- type rules, Signal Processing”, Signal Processing, Vol. 86, pp. 2771-2784. (2006).

[5] Zamani, M., Karimi,G. M., Sadati, N., Parniani, M., "Design of a fractional order PID controller for an AVR using particle swarm optimization", Control engineering practice, Vol. 17, issue 12. (2009).

[6] Monje, A., Blas, M., Vinagre, M., Feliu, V., and Chen, Y., "Tuning and auto-tuning of fractional order controllers for industry applications", Control engineering practice, Vol. 16, Issue 7, (2008).

[7] Lee, K., Kim, J., "Controller gain tuning of a simultaneous multi-axis PID control system using the Taguchi method", Control Engineering Practice, Vol. 8, Issue 8, (2000). 
[8] Ryckebusch, E. F., Craig, I. K. , "PID tuning for a multivariable plant using Taguchi-based methods", Proceedings of the 15th IFAC World Congress, Barcelona, Spain, (2002).

[9] Alfaro, V. M., Vilanova, R. , O. Arrieta "Maximum Sensitivity Based Robust Tuning for Two-Degree-ofFreedom Proportional-Integral Controllers", Industrial \& Engineering Chemistry Research, (2008).

[10] Santhakumar, M., Asokan, T., "A Self-Tuning Proportional-Integral-Derivative Controller for an Autonomous Underwater Vehicle, Based On Taguchi Method”, Journal of Computer Science, Vol. 6, Issue. 8, pp. 862-871, (2010).

[11] Accoto A, Carpino G, Sergi F, et al. , "Design and characterization of a novel high power series elastic actuator for a lower limb roboic orthosis", International Journal of Advanced Robotic Systems, (2013).

[12] Carpino,G., Accoto, D., Sergi, F., Luigi., T., N., Guglielmelli, E., "A Novel Compact Torsional Spring for Series Elastic Actuators for Assistive Wearable Robots", Journal of Mechanical Design, Vol., 134 ,Issue 12, (2012).

[13] Au, S., k., Dilworth, P., Herr, H., "An ankle-foot emulation system for the study of human walking biomechanics", International Conference on Robotics and Automation, Orlando, Florida, May, (2006).

[14] Kong, K., Bae, J., Tomizuka, M., "Control of rotary series elastic actuator for ideal force-mode actuation in humanrobot interaction applications", IEEE/ASME Transactions on Mechatronics, Vol. 14, Issue 1, (2009).

[15] Taylor, D., M., "A compact series elastic actuator for Bipedals with human-like dynamic performance", MSc Thesis, Crnegie Mellon University, (2011).

[16] Hutter, M.,Remy, C. D., Hoepflinger, M. A., Siegwart, R., "High compliant series elastic actuation for the robotic leg scarIETH", International Conference on Climbing and Walking Robots (CLAWAR), Paris, France, September 7, (2011).

[17] Misgeld, B., J., E., Geriach-Hahn, K., Ruschen, D., Popprapa, A., Leonhardt, S., "Control of adjustable compliant actuators", Machines, Vol. 2, Issue 2, pp. 134-157, (2014).

[18] Williamson, M. M., "Series Elastic Actuator", MSc Thesis, Massachusetts institute of Technology artificial intelligence laboratory, (1995).

[19] Vinagre, B. M., Podlubny, I., Petras, I., Chen, Y.Q. , "Using fractional order adjustment rules and fractional order reference models in Model Reference Adaptive Control”. Nonlinear Dynamics, Vol. 29, pp. 269-279, (2001).

[20] Monje, C.A, Chen, Y.Q. and Vinagre, B.M. , Book: Fractional order systems and controls fundamentals and applications, chapter 2, springer, (2010).

[21] Podlubny, I., "Fractional-order systems and $P I^{\lambda} D^{\mu}$ controllers". IEEE Transactions on Automatic Control, Vol. 44, no. 1, pp. 208-214, (1999).

[22] Petr'a s, I., "The fractional-order controllers: methods for their synthesis and application", Journal of Electrical Engineering, Vol. 50, , no. 9-10, pp. 284-288, (1999).

[23] Das, S., Saha, S., Das, Sh., Gupta, A., "On the Selection of Tuning Methodology of FOPID Controllers for the Control of Higher Order Processes", ISA Transactions, Vol. 50, Issue 3, pp: 376-388, (2011).

[24] Valerio, D., Costa, J., S., "Tuning of fractional PID controllers with Ziegler-Nichols-type rules", signal processing, Vol. 86, Issue 10, pp. 2771-2784, (2006).

[25] Hussain, M., Rajendran, Z., A., Kumar, Sh., Kumar, G., "Comparison of PID controller tuning methods with genetic algorithm for FOPTD system", Journal of engineering research and applications, Vol. 4, Issue 2, pp. 308$314,(2014)$ 